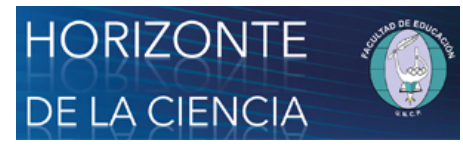

Horizonte de la Ciencia

ISSN: 2413-936X

horizontedelaciencia@uncp.edu.pe

Universidad Nacional del Centro del Perú

Perú

\title{
Las redes sociales y periodismo ciudadano en jóvenes de asentamientos humanos (AA. HH.) de Lima
}

Gómez Díaz, Rubén Luis; Chávez Cornejo, Margarita Angélica

Las redes sociales y periodismo ciudadano en jóvenes de asentamientos humanos (AA. HH.) de Lima

Horizonte de la Ciencia, vol. 10, núm. 18, 2020

Universidad Nacional del Centro del Perú, Perú

DOI: https://doi.org/10.26490/uncp.horizonteciencia.2020.18.398

Los autores otorgan el permiso a compartir y usar su trabajo manteniendo la autoría del mismo. Atribución no comercial (CC BY-NC) 4.0 


\section{Las redes sociales y periodismo ciudadano en jóvenes de asentamientos humanos} (AA. HH.) de Lima

Social networks and citizen journalism in young people from human settlements of Lima

As redes sociais e jornalismo cidadão em jovens de assentamentos humanos (AA. HH.) em Lima

Achkakunap tinkuyninkuna,malka willakuykunawan walalshwanlakuna nunanila taakunan Limaachu

NIYANKITSIRORI KENKITSABAERONTSI KOBAYENAKOTATSIRI KAMANTAJEITENERI

ATSIRIPE EBANKARIPAYENI SAINKANTARORI NANPITSIPE APATOKITACHARI ASHI IRIMASHIKI (KAIROMASHI).

Sangenarentsitsapage aike sangenare komantatsatagetiri iboingapage kara okaragitetagetanë timagantsipage irimakë

Rubén Luis Gómez Diaz Datos de los autores

Universidad César Vallejo, Perú

rgomezd@ucv.edu.pe

iD http://orcid.org/0000-0003-4703-2475

Margarita Angélica Chávez Cornejo

Universidad César Vallejo, Perú

angelicachavezc11@gmail.com

(iD) http://orcid.org/0000-0001-8956-9890
DOI: https://doi.org/10.26490/ uncp.horizonteciencia.2020.18.398

Recepción: 30 Marzo 2019 Aprobación: 20 Agosto 2019

Recepción: 30 Marzo 2019 Aprobación: 20 Agosto 2019

\section{Resumen:}

En los últimos diez años el periodismo tradicional sufrió un cambio significativo al realizarse la irrupción de los ciudadanos en el proceso informativo. Las nuevas tecnologías digitales y las redes sociales posibilitaron nuevas formas de acceso y participación de los jóvenes como nuevos actores sociales en la producción de información, ciudadanos que, con el uso de un teléfono móvil con cámara integrada, testigo de un hecho noticioso difunden no sólo videos y fotografías, sino también relatos desde el escenario de los hechos para el consumo del resto de la sociedad y en muchas ocasiones transmitidos por los medios de comunicación. El estudio aporta información importante para el entendimiento de las redes sociales y la relación con el periodismo ciudadano basado en la investigación de campo aplicado a una muestra de 80 jóvenes del A.H. San Antonio de Ate, siendo estos usuarios y consumidores de Internet y las redes sociales.

Palabras Clave: Redes sociales y periodismo ciudadano, niyankitsirori kenkitsabaerontsi kobayenakotatsiri, kamantajeiteneri, saikantarori nampitsipe, Sangenatsatagantsipage, komantatsatagantsipage.

\section{AbstraCt:}

In the last ten years, traditional journalism underwent a significant change when the emergence of citizens in the information process. The new digital technologies and social networks enabled new forms of access and participation of young people as new social actors in the production of information, citizens who with the use of a mobile phone with integrated camera, witness a

\section{NotAS DE AUTOR}

Datos Rubén Gómez Díaz. Investigador y docente de Ciencias de la Comunicación. Magister en Comunicación Interactiva con mención en Producción de los Audiovisual por la Universidad Nacional Federico Villareal, Ciudad de Lima. autores

Margarita Chávez Cornejo. Investigador y docente de Ciencias de la Comunicación. Magister en Administración de la Educación por la Universidad César Vallejo, Ciudad de Lima.

rgomezd@ucv.edu.pe 
news event broadcast not only videos and photographs, but also stories from the scene of events for the consumption of the rest of society and often transmitted by the media. The study provides important information for the understanding of social networks and the relationship with citizen journalism based on field research applied to a sample of 80 young people of human settlement San Antonio de Ate, being these users and consumers of Internet and social networks.

KEYWORDS: social networks and citizen journalism.

\section{Resumo:}

Nos últimos dez anos, o jornalismo tradicional sofreu uma mudança significativa quando a irrupção dos cidadãos ocorreu no processo de informação. As novas tecnologias digitais e redes sociais possibilitaram novas formas de acesso e participação dos jovens como novos atores sociais na produção da informação. Cidadãos que, com o uso de um celular com câmera integrada, testemunham um evento noticioso, difundem não só vídeos e fotografias, mas também relatos da cena dos fatos para o consumo do resto da sociedade e em muitas ocasiões transmitidas pela mídia. O estudo fornece informações importantes para a compreensão das redes sociais e o relacionamento com o jornalismo cidadão, com base em pesquisa de campo aplicada a uma amostra de 80 jovens do A.H. San Antonio de Ate, sendo esses usuários e consumidores da Internet e das redes sociais.

Palavras-chave: Redes sociais e jornalismo cidadão.

\section{LAS REDES SOCIALES Y PERIODISMO CIUDADANO EN JÓVENES DE ASENTAMIENTOS HUMANOS} (AA. HH.) DE LIMA

Los usos de las redes sociales son distintos y varían desde el entretenimiento hasta la expresión personal o los fines de transmisión de hechos noticiosos. Es evidente cómo estas herramientas han logrado protagonismo como plataformas de consumo de noticias a tal el punto de ser parte importante de los medios de comunicación y en muchos casos han sido fundamentales en la cobertura de noticiosos de última hora.

Parte de la publicación Reporte Digital in 2018, elaborado por las plataformas We are Social y Hootsuite, realizado en enero último sobre Social Media en Perú menciona que el país tiene 22 millones (68\%) de usuarios de internet, sobre los 32.3 millones de la población total. Los usuarios de redes sociales también alcanzan los 22 millones, y el crecimiento de consumo de las redes sociales el último año es de 10\%, mientras que los que ingresan a través de celulares crecieron $11 \%$.

En a la red social Facebook, de acuerdo al informe en el país existen 22 millones de usuarios actualmente. Cabe mencionar que $45 \%$ son mujeres y $55 \%$ son varones, además de que el $91 \%$ de usuarios se conecta a través de un smartphone.

El grupo más grande de usuarios lo constituyen los varones de entre 18 y 24 años (3.7 millones), le siguen los varones de entre 25 y 34 años (3.5 millones), y en el tercer grupo recién aparecen las mujeres de entre 18 y 24 años ( 3.1 millones). El cuarto lugar lo ocupan las mujeres de entre 25 y 34 ( 2.8 millones).

El uso de las redes sociales se ha convertido en una herramienta para comunicar un hecho que circunstancialmente ocurre cualquier lugar y el ciudadano que presencia reporta al resto de la sociedad. Recordemos el atentado contra las torres gemelas ocurridas un 11 de setiembre de 2001. El mundo se vio paralizado por esta tragedia y los protagonistas de la noticia eran los propios sobrevivientes y espectadores. Según Óscar Espiritusanto (2011), señala que Dan Gillmor padre del periodismo ciudadano o participativo fue clave para consolidar este el periodismo ciudadano como un fenómeno comunicacional imparable en la red. (pág.11). En consecuencia, los ciudadanos empiezan a organizarse y pasan de ser una masa anónima a constituirse en multitudes inteligentes.

El Perú, no es ajeno a esa realidad, sin embargo, el problema no radica en cómo se registra el evento, sino qué tratamiento le puedes dar a esa información sin llegar a causar zozobra en la población. En diciembre del 2016, cuando los pobladores de Huaycán, luego que circulara por el Facebook la existencia de un grupo de personas que estaban secuestrando a menores de edad para robarles sus órganos, originaron disturbios y destrozos, es más estuvieron a punto de linchar a dos parejas de encuestadores del Instituto Cuanto. (Perú $21,2016)$. 
El problema se da por inmediatez de la información, la noticia transmitida a través de las redes sociales carece de control y verificación siendo lanzada a la opinión pública sin ningún tipo de restricciones dando lugar a una serie de cambios conductuales en la ciudadanía. En el AA.HH. San Antonio del distrito de Ate los jóvenes en su gran mayoría portan diversos aparatos móviles, colgando en las redes sociales todo tipo de noticias que ellos obtienen a través de su dispositivo móvil, como son casos de bulling, agresiones físicas, actos e imágenes inmorales, así mismo, también son testigos de hechos fortuitos como accidentes automovilísticos, asalto y robo. Es en este contexto que el trabajo de investigación abordó las siguientes interrogantes ¿¿De qué manera el uso de las redes sociales influye en el periodismo ciudadano en jóvenes del AA. HH. San Antonio Ate-2017?

$\mathrm{Al}$ dar solución a las interrogantes se pudo determinar que las redes sociales directas como Facebook, twitter e instagram, así como las indirectas Blogs y Foros tienen una influencia significativa en el consumo y transmisión de informaciones por los jóvenes del AA.HH. San Antonio-Ate.

\section{Metodología}

\begin{tabular}{|l|l|l|}
\hline \multicolumn{3}{|c|}{ COEFICIENTE ALFA DE CRONBACH PARA LAS } \\
VARIABLES EN ESTUDIO \\
\hline Variable & $\begin{array}{l}\text { 2. Número de } \\
\text { Items }\end{array}$ & $\begin{array}{l}\text { Coeficiente alfa de } \\
\text { cronbach: a }\end{array}$ \\
\hline $\begin{array}{l}\text { Uso de redes } \\
\text { Sociales }\end{array}$ & 10 & 0.670 \\
\hline $\begin{array}{l}\text { Periodismo } \\
\text { Ciudadano }\end{array}$ & 10 & 0.722 \\
\hline
\end{tabular}

El estudio utilizado es el descriptivo, de enfoque cuantitativo de tipo transversal no experimental, la población, objeto de la investigación, está conformada por una total de 100 jóvenes entre hombres y mujeres del AA.HH. San Antonio de Ate y mediante el muestreo de aleatorio simple se determinó la muestra de estudio conformado por 80 jóvenes del AA.HH. San Antonio-Ate. Para el recojo de datos se usó como técnica la entrevista y como instrumento el cuestionario estructurado los cuales fueron validos a través de juicio de expertos y la confiabilidad conjunta de las variables periodismo ciudadano y uso de las redes sociales con el Alfa De Cronbach.

En la tabla observamos que el coeficiente Alfa de Cronbach para el Periodismo Ciudadano es mayor a 0.700; entonces concluimos que el instrumento de medición para esta variable es confiable.

\section{Resultados}

Para contrastar la hipótesis de que una variable independiente tenga efecto o influencia en la variable dependiente categórica está relacionada con la significancia de los coeficientes (estadísticamente diferente de cero) se empleó la prueba Ch-Cuadrada referido a la razón de verosimilitud del ajuste del modelo de regresión logística.

Contrastación de la hipótesis general

H1: El uso de las redes sociales influye significativamente en el periodismo ciudadano en jóvenes del AA.HH. San Antonio-Ate.2017.

Ho: El uso de las redes sociales no influye significativamente en el periodismo ciudadano en jóvenes del AA.HH. San Antonio-Ate.2017. 


\section{Taba 02 Tabla Test de la Chi-Cuadrada y su razón de Verosimilitud del Uso de Redes Sociales y Periodismo Ciudadano de los jóvenes en estudio}

\begin{tabular}{|c|c|c|c|}
\hline ESTADÍSTICOS & Valor & $\begin{array}{l}\text { Grados de } \\
\text { libertad }\end{array}$ & $\begin{array}{l}\text { Valor de } \\
\text { significancia, } \\
\text { p }\end{array}$ \\
\hline $\begin{array}{l}\text { VALOR CALCULADO DE CHI- } \\
\text { CUADRADO: } \square 20\end{array}$ & 8.593 & 1 & 0.003 \\
\hline $\begin{array}{l}\text { VALOR TABULAR DE CHI- } \\
\text { CUADRADO: } 02 \text { ( }\end{array}$ & 3.84 & & \\
\hline $\begin{array}{l}\text { RAZÓN DE VERISIMILITUDES } \\
\text { CHI-CUADRADA }\end{array}$ & 7.577 & 1 & 0.006 \\
\hline \multicolumn{4}{|c|}{$\begin{array}{c}\text { Como } \\
\text { p\# } 0.003 \# \\
\text { Fuente: Elaboración }\end{array}$} \\
\hline
\end{tabular}

para afirmar que el uso de las redes sociales influye significativamente en el periodismo ciudadano en jóvenes del AA.HH. San Antonio-Ate.2017; la influencia de la primera variable en la segunda queda sustentada en base a la significancia ( 0.006 \# \# 0.05 ) de la razón de verosimilitud de la prueba Chi-Cuadrada correspondiente (Ver Tabla 02)

\section{Sub Hipótesis $\mathrm{N}^{0} 1$}

H1: El uso de las redes sociales directas influye significativamente en el periodismo ciudadano en jóvenes del AA.HH. San Antonio-Ate.2017.

Ho: El uso de las redes sociales directas no influye significativamente en el periodismo ciudadano en jóvenes del AA.HH. San Antonio-Ate.2017.

\section{TABLA 03:}

Tabla de la Chi-Cuadrada y su razón de Verosimilitud del Uso de Redes Sociales Directa y Periodismo Ciudadano de los jóvenes en estudio

\begin{tabular}{|c|c|c|c|}
\hline ESTADÍSTICOS & Valor & $\begin{array}{l}\text { Grados de } \\
\text { libertad }\end{array}$ & $\begin{array}{l}\text { Valor de } \\
\text { significancia, } p\end{array}$ \\
\hline $\begin{array}{l}\text { VALOR CALCULADO DE CHI- } \\
\text { CUADRADO: } 120\end{array}$ & 19.558 & 2 & 0.000 \\
\hline $\begin{array}{l}\text { VALOR TABULAR DE CHI- } \\
\text { CUADRADO: } 12 \square\end{array}$ & 5.99 & & \\
\hline $\begin{array}{l}\text { RAZÓN DE VERISIMILITUDES } \\
\text { CHI-CUADRADA }\end{array}$ & 15.798 & 2 & 0.000 \\
\hline
\end{tabular}

Fuente: Elaboración propia

\# \# 0.05 , rechazamos H0 , es decir que existen evidencias estadísticas

para afirmar que el uso de las redes sociales directas influye significativamente en el periodismo ciudadano en jóvenes del

AA.HH. San Antonio-Ate.2017; la influencia de la primera variable en la segunda queda sustentada en base a la significancia 0.000 \# \# 0.05 ) de la razón de verosimilitud de la prueba Chi-Cuadrada correspondiente (Ver Tabla 03) 


\section{Sub Hipótesis $\mathrm{N}^{\circ} 2$}

H1: El uso de las redes sociales indirectas influye significativamente en el periodismo ciudadano de los jóvenes del AA.HH. San Antonio-Ate.2017.

Ho: El uso de las redes sociales indirectas no influye significativamente en el periodismo ciudadano de los jóvenes del AA.HH. San Antonio-Ate.2017.

\section{TABLA 04}

Test de la Chi-Cuadrada y su razón de Verosimilitud del Uso de Redes Sociales Indirecta y Periodismo Ciudadano de los jóvenes en estudio

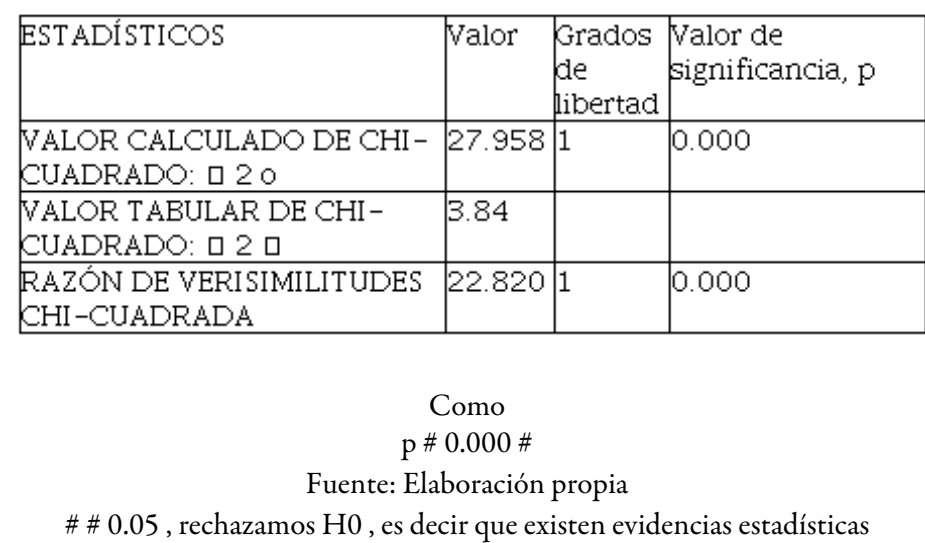

para afirmar que el uso de las redes sociales indirecta influye significativamente en el periodismo ciudadano en jóvenes del AA.HH. San Antonio-Ate.2017; la influencia de la primera variable en la segunda queda sustentada en base a la significancia ( 0.000 \# \# 0.05 ) de la razón de verosimilitud de la prueba Chi-Cuadrada correspondiente (Ver Tabla 04)

\section{Discusión}

La investigación tuvo como resultado en cuanto al uso de redes sociales de los jóvenes del AA.HH. San Antonio-Ate.2017 para reportar un hecho noticioso, el 21.25\% de los encuestados consideran que realizan de manera regular; y el $78.75 \%$, lo realiza de manera frecuente. Esto significa que el uso de las redes sociales influye significativamente en el periodismo ciudadano en jóvenes del AA.HH. San Antonio-Ate. Con respecto al uso de redes sociales directas de los Jóvenes del AA.HH. San Antonio-Ate.2017, el 11.25\% de los encuestados consideran que no hacen uso; el 13.75\%, de manera regular; y el 75\%, de manera frecuente, esto significa que el uso de las redes sociales directas influye significativamente en el periodismo ciudadano en jóvenes del AA.HH. San Antonio-Ate. Por último el uso de redes sociales indirectas por parte de los jóvenes del AA.HH. San Antonio-Ate.2017, el 13.75\% de los encuestados consideran que lo realizan de manera regular; y el $86.25 \%$, lo realiza con frecuencia, Esto nos permite afirmar que el uso de las redes sociales indirectas influye significativamente en el periodismo ciudadano de los jóvenes del AA.HH. San AntonioAte. 


\section{Conclusiones}

De acuerdo a los resultados estadísticos podemos afirmar que el uso de las redes sociales influye significativamente en el periodismo ciudadano en jóvenes del AA.HH. San Antonio- Ate. par esto ha sido determinante el desarrollo de la telefonía móvil que ha permitido el acceso y al reporte de hechos noticiosos democratizando aún más la información.

El tipo de redes sociales más usados por los jóvenes del AA.HH. San Antonio-Ate son los directos como el Facebook y twitter, mientras que las redes sociales indirectas son usadas, pero en menor porcentaje debido a que estos dependen una persona o de un grupo que contrala la información o tema de discusión como son los Blogs y Foros. Pero ambos influyen de manera significativa en el periodismo ciudadano.

Las redes sociales han provocado una transformación en la forma de transmitir la información otorgándole interactividad, inmediatez en tiempo record para reporta un hecho a través de un celular al ciudadano. Los periodistas recogen y clasifican estos hechos interactúa con las personas en busca de profundizar la información que reportan.

\section{ReFERENCIAS BIBLIOGRÁFICAS}

Amado, A. y Tarullo, R. (2015). Las redes sociales en la comunicación política:

Arnao, J. y Surpachin, M. (2016). Uso y abuso de las redes sociales digitales en adolescentes y jóvenes 2016. Recuperado de

Arroyo, G. \& Haro, M. (2016). Internet, redes sociales y periodismo ciudadano en los jóvenes universitarios de Latacunga (Tesis de Pregrado). Recuperado de: http://repositorio.utc.edu.ec/bitstream/27000/3825/1/TUTC-0107.pdf

Barrios, A. (2009). Los jóvenes y la red: usos y consumos de los nuevos medios en la sociedad de la información y la comunicación. Signo y pensamiento. 1 (56), 265-275.Recuperado de: http://www.scielo.org.co/pdf/signo/ v28n54/v28n54a17.pdf

Espiritusanto, O. \&Rodríguez, P. (2011). Periodismo Ciudadano: Evolución positiva de la comunicación. Barcelona, España: Editorial Ariel.

Los autores otorgan el permiso a compartir y usar su trabajo manteniendo la autoría del mismo. CC BY-NC 